\section{Efficacy of Sarilumab in hospitalized COVID-19 patients}

\author{
Vijairam Selvaraj ${ }^{1,2 *}$, Mohammad Saud Khan ${ }^{1,2}$, Kevin John John³, \\ Ajay Kumar Mishra ${ }^{4}$ \\ 'Division of Hospital Medicine, The Miriam Hospital, Providence, Rhode Island. \\ ${ }^{2}$ Warren Alpert Medical School of Brown University, Providence, Rhode Island. \\ ${ }^{3}$ Department of Medicine, Bangalore Baptist Hospital, Bangalore, Karnataka, India \\ ${ }^{4}$ Department of Internal Medicine, Saint Vincent Hospital, Worcester, \\ Massachusetts
}

Address for correspondence:

Vijairam Selvaraj MD, MPH, FACP. The Miriam Hospital, Warren Alpert Medical School of Brown University, Providence, RI 02906.

Email: vijairam.selvaraj@lifespan.org.

Ph: 413-271-0421. ORCID: 0000-0002-8507-9891

How to cite this article: Selvaraj V, Khan M S, John K, Mishra AK. Efficacy of Sarilumab in hospitalized COVID-19 patients. G Med Sci. 2021; 2(3): 050-052.

https://www.doi.org/10.46766/thegms.intmed.21062302

Copyright: (c) 2021 Vijairam Selvaraj, Mohammad Saud Khan, Kevin John, Ajay Kumar Mishra. This is an Open Access article distributed under the Creative Commons Attribution License, which permits unrestricted use, distribution, and reproduction in any medium, provided the original work is properly cited.

\begin{abstract}
Interest in modulating the immune response beyond glucocorticoids has existed since the COVID-19 pandemic began. Recent trials have shown promising results with IL-6 inhibitors such as tocilizumab although similar data on Sarilumab is lacking. In this report, we analyze the current evidence on Sarilumab in hospitalized COVID-19 patients to evaluate its efficacy.
\end{abstract}

Keywords: SARS-CoV2, COVID-19, Sarilumab, meta-analysis, IL-6

COVID-19 is associated with hyper inflammation, especially in severe cases. To date, only glucocorticoids (mainly dexamethasone) have been shown to reduce mortality in hypoxemic COVID-19 patients [1]. Literature has shown that several cytokines and chemokines may be involved in the hyperinflammatory response. Of these, IL-6 plays a central role by promoting proliferation of myeloid precursor cells, synthesis of $\mathrm{C}$ reactive proteins and suppression of T-lymphocytes, dendritic cells, and macrophages [2]. This results in a decreased ability of the immune system to clear SARS-CoV2. A meta-analysis of 23 trials showed that IL-6 concentrations were higher in COVID-19 patients with severe disease than mild disease [3]. Elevated IL-6 levels have also been associated with progression to mechanical ventilation and death [3, 4].

Sarilumab is a human monoclonal, IL-6 antibody that is approved for the treatment of moderate to severe rheumatoid arthritis. It works by inhibiting both soluble and membrane-bound forms of IL- 6 receptors, thereby suppressing proinflammatory pathways. Like tocilizumab, sarilumab is thought to suppress the hyperinflammatory syndrome induced by chimeric antigen receptor $\mathrm{T}$-cells. Limited supplies of tocilizumab have led to use of sarilumab as an alternate agent to treat COVID-19 patients. Recent trials on tocilizumab use showed improved survival, probability of discharge from hospital alive by 28 days and reduced probability of progression to invasive mechanical ventilation [5, 6]. However, uncertainty remains regarding the role of sarilumab in COVID-19 patients. The findings presented by Lescure and colleagues in The Lancet Respiratory Medicine are therefore a welcome addition to this topic [7]. In this report, we sought to examine the current evidence on the efficacy of sarilumab in hospitalized COVID-19 patients considering the recent trials.

We searched MEDLINE and preprint databases from March 1st, 2020, to March 10th, 2021 to identify all relevant articles using the following search terms: ("SARS-CoV2" OR "COVID-19") AND ("sarilumab" OR "IL-6" OR "AntiIL6"). We also searched the bibliographies of relevant articles. Inclusion criteria were: (1) Clinical trial, cohort 
studies, and case-control studies; (2) Studies included patients with confirmed COVID-19 infection who received sarilumab and were compared to patients with confirmed COVID-19 infection who did not receive sarilumab; (3) Desired outcomes were reported in the study. Results were limited to humans and the English language. Outcomes of interest were mortality at 28 days and progression to mechanical ventilation. The Review Manager software (version 5.4.1, The Cochrane Collaboration) was used for statistical analysis. Mantel-Haenszel risk ratios and 95\% confidence intervals (CIs) were calculated. I2 index was used for heterogeneity estimation. The meta-analysis was performed with a random effects model.

The initial database query revealed 86 articles. After careful review, only 8 studies met the inclusion criteria although 4 studies did not delineate results between different IL- 6 inhibitors. Hence, only 4 studies ( 3 randomized controlled trials, 1 observational studies) were included for analysis [6-9]. A total of 1,148 patients were included, of which 590 patients received sarilumab and 558 received standard care or placebo. In the mortality analysis, 4 studies were included [6-9]. A total of 96 deaths out of 590 participants were reported in the sarilumab arm compared to 166 deaths out of 558 participants in the standard care or placebo arm. Pooled analysis of mortality outcome at 28 days favoured treatment with sarilumab although did not reach statistical significance (RR 0.83, 95\% CI 0.56-1.22, $\mathrm{p}=0.34)$.

The results of the present analysis show possible mortality benefits associated with the use of sarilumab in patients with COVID-19 infection, even though results were not statistically significant. Certain limitations exist with our meta-analysis. First, one of the included studies was an observational study. Second, sample sizes were small in the included studies, much smaller than the tocilizumab trials. Third, there was heterogeneity in steroid use and standard of care across different studies. Fourth, RCTs were open label in design and among them, one only reported preliminary results. This would predispose to performance and selective reporting biases.

Given limited availability of these drugs, further data is necessary on selection of patients that are most likely to benefit along with timing of intervention. Prior trials on IL- 6 inhibitors have used CRP levels $>75 \mathrm{mg} / \mathrm{L}$ or initiation of organ support as criteria [5, 6]. Though there was varying use of glucocorticoids in this meta-analysis, recent guidelines by National Institutes of Health (NIH) recommended using IL-6 inhibitors in combination with glucocorticoids in hypoxemic patients [10]. In conclusion, present data on sarilumab use is promising although larger trials are necessary to determine if there is benefit from IL-6 inhibition in general or whether specific medications have a differential effect. Further data is also expected from an ongoing trial (NCT04315298) with a greater sample size.

\section{Author contributions}

VS and MSK had access to the data and were primarily involved in writing the manuscript. KJJ and AKM were involved in revising the manuscript and making critical changes.

\section{Conflict of Interest: None}

\section{Funding: None}

\section{References}

1. RECOVERY Collaborative Group, Horby P, Lim WS, Emberson JR, et al. Dexamethasone in Hospitalized Patients with Covid-19. N Engl J Med. 2021 Feb 25;384(8):693-704. doi: 10.1056/NEJMoa2021436. Epub 2020 Jul 17. PMID: 32678530; PMCID: PMC7383595.

2. Datta C, Bhattacharjee A. Cytokine Storm and Its Implication in Coronavirus Disease 2019 (COVID-19). J Clin Immunol. 2020 Sept 18.

3. Zhu J, Pang J, Ji P, et al. Elevated interleukin-6 is associated with severity of COVID-19: a meta-analysis. J Med Virol 2020; published online May 29. https://doi.org/10.1002/jmv.26085.

4. Herold T, Jurinovic V, Arnreich C, et al. Elevated levels of IL-6 and CRP predict the need for mechanical ventilation in COVID-19. J Allergy Clin Immunol 2020;146(1):128-136.e4.

5. Horby PW, Pessoa-Amorim G, Peto L, et al. Tocilizumab in patients admitted to hospital with COVID-19 (RECOVERY): preliminary results of a randomised, controlled, open-label, platform trial. medRxiv 2021: Available at: https://doi. org/10.1101/2021.02.11.21249258 [Preprint 11 February 2021].

6. REMAP-CAP Investigators, Gordon AC, Mouncey PR, Al-Beidh F, et al. Interleukin-6 Receptor Antagonists in Critically Ill Patients with Covid-19. N Engl J Med. 2021 Feb 25. doi: 10.1056/ NEJMoa2100433. Epub ahead of print. PMID: 33631065 .

7. Lescure FX, Honda H, Fowler RA, et al.; Sarilumab COVID-19 Global Study Group. Sarilumab in patients admitted to hospital with severe or 
critical COVID-19: a randomised, double-blind, placebo-controlled, phase 3 trial. Lancet Respir Med. 2021 Mar 4:S2213-2600(21)00099-0. doi: 10.1016/S2213-2600(21)00099-0. Epub ahead of print. PMID: 33676590.

8. Della-Torre E, Campochiaro C, Cavalli G, et al.; SARI-RAF Study Group; SARI-RAF Study Group members. Interleukin-6 blockade with sarilumab in severe COVID-19 pneumonia with systemic hyperinflammation: an open-label cohort study. Ann Rheum Dis. 2020 Oct;79(10):1277-1285. doi: 10.1136/annrheumdis-2020-218122. Epub 2020 Jul 3. PMID: 32620597; PMCID: PMC7509526.
9. Sanofi: Press Releases, Sanofi and Regeneron provide update on U.S. Phase $2 / 3$ adaptive-designed trial in hospitalized COVID-19 patients. https:// www.sanofi.com/en/media-room/press-releases/2020/2020-04-27-12-58-00. Accessed March $18,2021$.

10. National Institutes of Health (NIH) COVID-19 Treatment Guidelines. The COVID-19 Treatment Guidelines Panel's Statement on the Use of Tocilizumab for the Treatment of COVID-19 https:// www.covid19treatmentguidelines.nih.gov/statement-on-tocilizumab/ Accessed March 10, 2021.

\begin{tabular}{|c|c|c|c|c|c|c|c|c|}
\hline \multirow[b]{2}{*}{ Study or Subgroup } & \multicolumn{2}{|c|}{ Sarilumab } & \multicolumn{2}{|c|}{ Standard Care/Placebo } & \multirow[b]{2}{*}{ Weight } & \multirow{2}{*}{$\begin{array}{c}\text { Risk Ratio } \\
\mathrm{M}-\mathrm{H}, \text { Random, } 95 \% \mathrm{Cl}\end{array}$} & \multirow{2}{*}{\multicolumn{2}{|c|}{$\begin{array}{c}\text { Risk Ratio } \\
\mathrm{M}-\mathrm{H}, \text { Random, } 95 \% \mathrm{Cl}\end{array}$}} \\
\hline & Events & Total & Events & Total & & & & \\
\hline REMAP-CAP & 10 & 48 & 142 & 402 & $34.8 \%$ & $0.59[0.33,1.04]$ & & $\rightarrow-1$ \\
\hline Sarilumab CovID-19 & 30 & 332 & 7 & 84 & $20.9 \%$ & $1.08[0.49,2.38]$ & & \\
\hline U.S. Kevzara & 54 & 182 & 12 & 44 & $38.2 \%$ & $1.09[0.64,1.85]$ & & 一 \\
\hline Della-Torre et al. & 2 & 28 & 5 & 28 & $6.1 \%$ & $0.40[0.08,1.89]$ & & \\
\hline Total $(95 \% \mathrm{Cl})$ & & 590 & & 558 & $100.0 \%$ & $0.83[0.56,1.22]$ & & \\
\hline Total events & 96 & & 166 & & & & & \\
\hline \multicolumn{6}{|c|}{$\begin{array}{l}\text { Heterogeneity. Tau }{ }^{2}=0.03 ; C^{2} i^{2}=3.71, d f=3(P=0.29) ;\left.\right|^{2}=19 \% \\
\text { Test for overall effect: } Z=0.95(P=0.34)\end{array}$} & & 0.01 & $\begin{array}{cccc}0.1 & 10 & 100 \\
\text { Favours Sarilumab } & \text { Favours Standard Care/Placebo }\end{array}$ \\
\hline
\end{tabular}

Figure 1: Forest plot showing all-cause mortality outcome in patients that received sarilumab compared to standard care or placebo. 